\title{
Calculation of intergranular stresses based on a large-strain viscoplastic self-consistent polycrystal model
}

\author{
R A Lebensohn $\dagger$, P A Turner†, J W Signorelli†, G R Canova $\ddagger \|$ and \\ C N Tomé $\oint$ \\ $\dagger$ Instituto de Física Rosario (UNR-CONICET), 27 Febrero 210 Bis, 2000 Rosario, Argentina \\ $\ddagger$ GPM2, ENSPG, Domaine Universitaire, BP 46, 38402 St Martin d'Heres Cedex, France \\ $\S$ Center for Materials Science, Los Alamos National Laboratory, Los Alamos, NM 87545, USA
}

Received 22 January 1998, accepted for publication 19 April 1998

\begin{abstract}
We present here an extension of the viscoplastic self-consistent (VPSC) polycrystal model for the calculation of the intergranular Cauchy stresses in an aggregate. This method, which is based on the self-consistent treatment of incompressible aggregates proposed in 1987 by Molinari et al, is formulated using the inclusion formalism and full anisotropy is incorporated into it. The complete stress state in the grains is obtained by computing the deviatoric and the hydrostatic local deviations with respect to the overall corresponding magnitudes applied to the polycrystal. The extended VPSC model, followed by an elastic self-consistent unloading, is used to obtain the intergranular residual strains in the aggregate after large plastic deformation. The texture evolution and the hardening of the material are explicitly taken into account in the model. As an application, the model is used to predict intergranular residual states in Incoloy-800 plate after uniaxial deformation.
\end{abstract}

\section{Introduction}

Residual stresses induced by cold working of polycrystalline materials usually influence the ulterior mechanical response and properties of the aggregate, such as the fracture or the creep characteristics. In this work we propose a method for calculating intergranular stresses in aggregates after cold working. Elasto-plastic models of polycrystals vary in the degree of sophistication used to describe the interaction of each grain with its surroundings. The simplest is probably Lin's model [1], which involves the assumption that the sum of the elastic and the plastic strain components are the same in every grain. Kröner [2] and the Budiansky-Wu model [3] use the solution of the inclusion problem [4] to allow for elastic accommodation of strain in the matrix, but this method is very rigid and converges to Lin's model when the plastic deformation is of the order of the elastic deformation. A more sophisticated model, also based on the inclusion formalism, is that proposed by Hill [5], which allows for elasto-plastic accommodation of strain by the matrix. In a recent review paper, Molinari et al [6] argue that Hill's model is also characterized by a stiff interaction between grain and matrix because it is based on an implicit secant approach. They propose instead an elasto-visco-plastic model based on a tangent approach.

In addition to the stiffness of the interaction, when dealing with large strains one is inevitably concerned with the reorientation of the individual grains. Consideration of

|| Deceased on 28 July 1997.

0965-0393/98/040447+19\$19.50 (C) 1998 IOP Publishing Ltd 
such an effect requires the incorporation of the spin of the stress tensor and adds another dimension of complexity to the model (see for example Iwakuma and Nemat-Nasser [7]). More recent work by Berveiller et al [8] and Krier et al [9] predicts the evolution of intergranular stresses within a large-strain elasto-plastic self-consistent (EPSC) formulation. The numerical implementation of these EPSC formulations requires long computing times, even to reach moderate degrees of deformation, because the strain increments have to be kept small in order to follow the evolution of stress across the yield surface of each grain. In this work we argue that it is not necessary to keep track of the elastic component when modelling large-strain deformation $(\epsilon>2 \%)$, since once the grains are in the plastic regime it is the evolution of the single-crystal yield surface that controls the stress. As a matter of fact, the latter assumption is implicit in all large-strain polycrystal models of texture development. One immediate advantage of this approach is that it is easy to follow the stress evolution associated with the reorientation of the grains. One problem, however, is that since plasticity is a function of only the deviatoric stress component, the approach does not provide information about the hydrostatic stress in the grains.

Van Acker et al [10] resolve such conflict by assuming the pressure to be the same in all grains and equal to the overall hydrostatic stress, which follows from the boundary conditions of the test. Their model, however, is based on the Taylor approach for describing the plastic deformation of the aggregate, followed by a Voigt model to describe the elastic unloading of the specimen. The condition of equal strain in all the grains, however, imposes severe restrictions on the stress and tends to disregard the equilibrium condition and the grain anisotropy. For this reason, in this work we adopt the idea of Van Acker et al but implement it within a scheme consisting of a self-consistent viscoplastic loading followed by self-consistent elastic unloading. This approach does not require any assumptions on the hydrostatic pressure distribution prior to unloading, since that is a result of the extended VPSC model. In addition, the self-consistent (SC) formulation allows us to consider the relative anisotropy of grain and matrix, within both the plastic and the elastic regimes. The self-consistency of the solution requires a more subtle treatment of the hydrostatic component, which is not uniform in our formulation.

The mechanical properties of a polycrystalline aggregate are heterogeneous, i.e. they vary from grain to grain. The SC models for the prediction of the mechanical behaviour of polycrystals are based on treating each grain or cluster of grains as local inhomogeneities embedded in an homogeneous equivalent medium (HEM) having the same overall response as the polycrystal (see for example Molinari et al [11], Lebensohn and Tomé [12]). This approach requires us to solve the interaction of each grain (or cluster of grains) with the HEM. The self-consistency of the solution follows from requiring the assumed properties of the HEM to be consistent with the spatial averages of the local states. Since the inhomogeneity problem can be formulated in terms of an equivalent inclusion problem [13], we shall refer to the grains (inhomogeneities) as inclusions in what follows.

Examples of SC viscoplastic polycrystal models include those developed by Hutchinson [14] to study thermal creep, by Molinari et al [11] and Lebensohn and Tomé [12] to analyse texture development associated with plastic forming, and by Tomé et al [15] to model linear creep and growth. All viscoplastic models have in common the fact that the medium is incompressible: the local and the overall response are independent of the hydrostatic pressure component, and deformation takes place without volume change. Consequently, there is an indeterminacy in the mathematical relation between Cauchy stress and strain rate. Since the Eshelby solution relies on the existence of a stiffness which determines a linear and one-to-one relation between the six independent components of the Cauchy stress and the components of the strain (or strain rate) tensor, the classical Eshelby solution cannot be 
directly applied to the viscoplastic inclusion.

To overcome this singular behaviour, Hutchinson [14] and Lebensohn and Tomé [12] assumed a small (but non-zero) fictitious compressibility in the medium and replaced the elastic stiffness by this 'slightly compressible' viscoplastic stiffness in Eshelby's solution. Molinari et al [11], on the other hand, applied a different strategy to find the exact solution of the viscoplastic inclusion problem. They solved the equilibrium equation by separating the deviatoric and the hydrostatic components of the stress, and using the incompressibility condition as an extra equation to determine the pressure, which allows one to calculate the local Cauchy stress associated with plastically deforming aggregates. Once the Cauchy stresses are known, it is possible to simulate the elastic unloading of the aggregate and evaluate the associated residual stresses.

In section 2 of this work we cast the theory of incompressible viscoplastic aggregates of Molinari et al [11] within the framework of the incompressible inclusion, derive closed integrals for the calculation of local Cauchy stresses, and propose a method for transferring from the viscoplastic to the elastic regime before unloading the aggregate. The anisotropy of the inclusion and of the surrounding medium are explicitly included in the formulation. In section 3 we present a simple application of the model, since the emphasis of the paper is on the method itself and not on a detailed analysis of a particular system. We predict the distribution of intergranular residual stresses associated with cold working of the austenitic alloy Incoloy-800 used in the fabrication of heat exchangers. Finally, in section 4 we draw some general conclusions concerning the validity and limitations of this approach. To enable the reader to follow the flow of ideas more easily, we have tried to reproduce the equations associated with the method in some detail. This implies some degree of repetition (but also further expansion and derivation of explicit expressions) of the previous work of Molinari et al [11] and Lebensohn and Tomé [12]. We feel that it is important to collect together all these elements in a single paper which is both comprehensive and sufficiently detailed for easy implementation.

\section{Extension of the viscoplastic self-consistent model}

\subsection{Viscoplastic inclusion problem}

In this section we combine elements of the formulations presented by Molinari et al [11] and Lebensohn and Tomé [12] for viscoplastic aggregates and repeat the relevant equations. The stress and the strain rate in the domain of the grains are homogeneous, as a result of linearizing the constitutive response and assuming ellipsoidal grain shape. Repeated indices indicate summation, bold letters second- and fourth-order tensors, and the symbol ':' denotes the double-contracted tensor product.

At the grain level (i.e. the viscoplastic inclusion), the strain rate is assumed to be related to the deviatoric stress through a nonlinear rate-sensitive equation:

$$
\dot{\epsilon}_{i j}=\sum_{s} m_{i j}^{s} \dot{\gamma}^{s}=\dot{\gamma}_{0} \sum_{s} m_{i j}^{s}\left(\frac{\boldsymbol{m}^{s}: \boldsymbol{\sigma}^{\prime}}{\tau_{0}^{s}}\right)^{n}
$$

where $\boldsymbol{m}^{s}, \gamma^{s}$ and $\tau_{0}^{s}$ are, respectively, the Schmid tensor, the single-shear rate and a threshold stress (that can be identified with the critical resolved shear stress (CRSS) of slip system $(s), n$ is the reciprocal of the rate sensitivity, and $\dot{\gamma}_{0}$ is a reference rate. The sum runs over the complete set of active slip systems. The constitutive equation may be expressed using either the secant or the tangent form:

$$
\dot{\epsilon}=M^{c(\mathrm{sec})}: \sigma^{\prime}
$$




$$
\dot{\epsilon}=M^{c(\tan )}: \sigma^{\prime}+\dot{\epsilon}^{0}
$$

where the local secant compliance of each individual grain is defined by:

$$
M_{i j k l}^{c(\mathrm{sec})}=\dot{\gamma}_{0} \sum_{s} \frac{m_{i j}^{s} m_{k l}^{s}}{\tau_{o}^{s}}\left(\frac{\boldsymbol{m}^{s}: \boldsymbol{\sigma}^{\prime}}{\tau_{0}^{s}}\right)^{n-1} .
$$

The tangent form (equation $(2 b)$ ) follows from taking a Taylor expansion around a fixed value of stress, and $\dot{\epsilon}^{0}$ is a back-extrapolated term. From equation (1) it is easy to see that the secant and tangent compliance of the single crystal are related through:

$$
M_{i j k l}^{c(\mathrm{tan})}=\frac{\partial \dot{\epsilon}_{i j}}{\partial \sigma_{k l}^{\prime}}=n M_{i j k l}^{c(\mathrm{sec})} \text {. }
$$

Secant and tangent constitutive laws of the form of (2) can be assumed to describe the overall response of the polycrystal (i.e. the viscoplastic HEM):

$$
\begin{aligned}
& \overline{\dot{\epsilon}}=M^{(\mathrm{sec})}: \bar{\sigma}^{\prime} \\
& \overline{\dot{\epsilon}}=M^{(\mathrm{tan})}: \bar{\sigma}^{\prime}+\bar{\epsilon}^{0}
\end{aligned}
$$

where $M^{(\mathrm{sec})}$ and $\boldsymbol{M}^{\text {(tan) }}$ are the overall (polycrystal) secant and tangent compliance tensors, while $\overline{\dot{\epsilon}}$ and $\bar{\sigma}^{\prime}$ are the overall strain-rate and stress tensors, respectively and $\dot{\epsilon}^{0}$ is the macroscopic back-extrapolated term. Hutchinson [14] proves that the tangent and secant moduli of the aggregate are simply proportional to each other, as they are in the single crystal:

$$
M_{i j k l}^{(\mathrm{tan})}=\frac{\partial \overline{\dot{\epsilon}}_{i j}}{\partial \bar{\sigma}_{k l}^{\prime}}=n M_{i j k l}^{(\mathrm{sec})} .
$$

For what follows it is convenient to rewrite the strain rate in the inclusion (equation $(2 b)$ ) as:

$$
\dot{\epsilon}=\left(M^{(\tan )}: \sigma^{\prime}+\dot{\epsilon}^{0}\right)+\dot{\epsilon}^{*}
$$

where:

$$
\dot{\epsilon}^{*}=\left(M^{c(\tan )}-M^{(\tan )}\right): \sigma^{\prime}+\left(\dot{\epsilon}^{0}-\dot{\epsilon}^{0}\right)
$$

is a fictitious strain rate that allows one to regard the inhomogeneity as an inclusion with the same properties as the effective medium. Defining the local deviations of the stress and strain rate from the overall values:

$$
\begin{aligned}
& \tilde{\boldsymbol{\sigma}}^{\prime}=\sigma^{\prime}-\overline{\boldsymbol{\sigma}}^{\prime} \\
& \tilde{\dot{\epsilon}}=\dot{\epsilon}-\overline{\dot{\epsilon}}
\end{aligned}
$$

and combining equations (5), (7) and (9) we obtain:

$$
\tilde{\dot{\epsilon}}-\dot{\epsilon}^{*}=M^{(\tan )}: \tilde{\sigma}^{\prime} .
$$

Equation (10) can be formally inverted to give:

$$
\tilde{\boldsymbol{\sigma}}^{\prime}=\boldsymbol{L}^{(\tan )}:\left[\tilde{\tilde{\epsilon}}-\dot{\epsilon}^{*}\right]
$$

where $\boldsymbol{L}^{(\tan )}$ is the fourth-order tangent viscoplastic stiffness tensor. In appendix A we show how to calculate $\boldsymbol{L}^{(\tan )}$ from $\boldsymbol{M}^{(\mathrm{tan})}$ using a deviatoric space representation. This procedure is different from the approximate penalty method used by Hutchinson [14] and Lebensohn and Tomé [12], involving the assumption of a small but non-zero compressibility. 
Following Molinari et al [11] we decompose the stress into its deviatoric and pressure components, and express the equilibrium equation as:

$$
\sigma_{i j, j}=\tilde{\sigma}_{i j, j}=\tilde{\sigma}_{i j, j}^{\prime}+\tilde{p}_{, i}=0
$$

where $\tilde{p}$ is the local deviation in pressure from the macroscopic pressure. Expressing the strain-rate deviations in terms of displacement-rate deviations:

$$
\tilde{\dot{\epsilon}}_{k l}=\frac{1}{2}\left(\tilde{\dot{u}}_{k, l}+\tilde{\dot{u}}_{l, k}\right)
$$

and replacing the constitutive equation (11) in the equilibrium equation (12) gives

$$
L_{i j k l}^{(\tan )}\left(\tilde{\dot{u}}_{k, l j}(\overline{\boldsymbol{r}})-\dot{\epsilon}_{k l, j}^{*}(\overline{\boldsymbol{r}})\right)+\tilde{p}_{, i}(\overline{\boldsymbol{r}})=0 .
$$

Equation (14) marks a general dependence upon the position. However, for the case under consideration, the stress, the strain rate and the eigenstrain $\dot{\epsilon}^{*}$ are uniform inside the domain of the ellipsoidal inclusion. As a consequence, $\tilde{\dot{u}}_{k, l}$ and $\tilde{\sigma}_{k l}$ do not depend on $\overline{\boldsymbol{r}}$ inside the inclusion domain. Adding the incompressibility condition, the complete set of differential equations to be solved is:

$$
L_{i j k l}^{(\tan )} \tilde{\dot{u}}_{k, l j}(\overline{\boldsymbol{r}})+\tilde{p}_{, i}(\overline{\boldsymbol{r}})+f_{i}(\overline{\boldsymbol{r}})=0 \quad \tilde{\dot{u}}_{k, k}(\overline{\boldsymbol{r}})=0
$$

where $f_{i}(\overline{\boldsymbol{r}})$ is a fictitious force given by:

$$
f_{i}(\overline{\boldsymbol{r}})=\Delta_{i j, j}(\overline{\boldsymbol{r}})
$$

and

$$
\Delta_{i j}(\overline{\boldsymbol{r}})=-L_{i j k l}^{(\tan )} \dot{\epsilon}_{k l}^{*} .
$$

Expression (15) is a system of four differential equations with four unknowns: the velocity field $\tilde{\dot{u}}_{i}(\overline{\boldsymbol{r}})(i=1,2,3)$ and the pressure field $\tilde{p}(\overline{\boldsymbol{r}})$. This system was originally solved by Molinari et al [11] using the Green function approach. While the latter authors did not make explicit use of the pressure in their application, their formal solution of the incompressible inclusion problem represents an important advance over the approximate solution that results from assuming a small compressibility. In appendix B we reproduce their derivation in order to obtain explicit integral solutions for a fully anisotropic medium. According to equation (B9), the strain-rate deviation can be written as:

$$
\tilde{\dot{\epsilon}}=S^{(\mathrm{vp})}: \dot{\epsilon}^{*}
$$

where:

$$
S_{i j k l}^{(\mathrm{vp})}=\frac{1}{4}\left(T_{i j m n}+T_{j i m n}+T_{i j n m}+T_{j i n m}\right) L_{m n k l}^{(\mathrm{tan})}
$$

is the viscoplastic Eshelby tensor and the tensor $\boldsymbol{T}$ is given by equation (B11). Replacing (18) in (10) gives a relation between the stress and the strain deviations in the inclusion:

$$
\tilde{\dot{\epsilon}}=-\tilde{M}^{(\mathrm{vp})}: \tilde{\boldsymbol{\sigma}}^{\prime} .
$$

In this interaction equation, $\tilde{M}^{(\mathrm{vp})}$ is the interaction tensor, given by:

$$
\tilde{\boldsymbol{M}}^{(\mathrm{vp})}=n\left(\boldsymbol{I}-\boldsymbol{S}^{(\mathrm{vp})}\right)^{-1}: \boldsymbol{S}^{(\mathrm{vp})}: \boldsymbol{M}^{(\mathrm{sec})} \quad \text { (tangent approximation). }
$$

In equation (21) we have explicitly used the relation between the secant and the tangent compliance (equation (6)), but the derivation is consistent with using the tangent constitutive law (equation $(5 b))$. On the other hand, if the secant approximation (equation $(5 a)$ ) is 
assumed to describe the interaction between the inclusion and the HEM, the interaction tensor to be used in (20) is affected by a factor $n$ [12]:

$$
\tilde{\boldsymbol{M}}^{(\mathrm{vp})}=\left(\boldsymbol{I}-\boldsymbol{S}^{(\mathrm{vp})}\right)^{-1}: \boldsymbol{S}^{(\mathrm{vp})}: \boldsymbol{M}^{(\mathrm{sec})} \quad \text { (secant approximation). }
$$

The differences in the predicted residual strains which arise from using the secant or the tangent approximation will be discussed later in section 3 .

Another magnitude of interest which follows from solving the viscoplastic inclusion problem is the viscoplastic localization tensor $\boldsymbol{B}^{c(\mathrm{vp})}$, which determines a linear relation between the stress in the inclusion and the overall stress. It follows from replacing $(9 a)$ in the interaction equation (20) that:

$$
\sigma^{\prime}=B^{c(\mathrm{vp})}: \bar{\sigma}^{\prime}
$$

where:

$$
\boldsymbol{B}^{c(\mathrm{vp})}=\left(\boldsymbol{M}^{c(\mathrm{sec})}+\tilde{\boldsymbol{M}}^{(\mathrm{vp})}\right)^{-1}:\left(\boldsymbol{M}^{(\mathrm{sec})}+\tilde{\boldsymbol{M}}^{(\mathrm{vp})}\right) .
$$

The second part of the incompressible inclusion problem addresses the pressure component, and the pressure deviation is given by equation (B13) as:

$$
\tilde{p}=\boldsymbol{P}: \boldsymbol{L}^{(\tan )}: \dot{\epsilon}^{*}=\boldsymbol{P}: \boldsymbol{L}^{(\tan )}: \boldsymbol{S}^{(\mathrm{vp})^{-1}}: \tilde{\dot{\epsilon}}
$$

where the second-order tensor $\boldsymbol{P}$ is given by (B14). Observe that the expression (25) allows us to calculate the local deviation in pressure when the strain-rate deviation is known. Equation (25) may be rewritten in the form of a localization for the pressure as:

$$
p=\left(1+\frac{\boldsymbol{P}: \boldsymbol{L}^{(\mathrm{tan})}: \boldsymbol{S}^{(\mathrm{vp})^{-1}}: \tilde{\dot{\epsilon}}}{\bar{p}}\right) \bar{p}
$$

which allows us to combine equations (23) and (26) into an extended localization equation for the Cauchy stress:

$$
\boldsymbol{\sigma}=\boldsymbol{B}^{c(\mathrm{xvp})}: \overline{\boldsymbol{\sigma}}
$$

When the tensors are represented in the symmetric basis described in appendix A this amounts to adding a non-zero element $B_{66}^{c}$ to the $5 \times 5$ matrix representing the localization tensor $\boldsymbol{B}^{c(\mathrm{vp})}$.

\subsection{Calculation of intergranular strains and stresses}

The latter solution for the pressure can be included in a viscoplastic self-consistent polycrystal model for calculating local Cauchy stresses. The self-consistent condition that the overall secant compliance has to fulfil is [12]:

$$
\boldsymbol{M}^{(\mathrm{sec})}=\left\langle\boldsymbol{M}^{c(\mathrm{sec})}: \boldsymbol{B}^{c(\mathrm{vp})}\right\rangle .
$$

The symbol \langle\rangle denotes a polycrystal average, where each orientation is weighted by the volume fraction that it represents. Once equation (28) is solved iteratively (i.e. the converged values of the overall secant and tangent compliance are found) the complete overall state can be obtained and the resulting interaction tensor can be used to obtain the local strain rate and deviatoric stress by solving the interaction equation (20) for each grain. Once $\tilde{\dot{\epsilon}}$ is known the local deviation in pressure $\tilde{p}$ can be obtained from (25).

Usually, the overall applied boundary conditions contain information about at least one diagonal component of the macroscopic Cauchy stress $\dagger$. As a consequence, the overall

$\dagger$ For example: (i) channel die, with the rolling direction along: $x_{1}: \bar{\sigma}_{11}=0$; (ii) uniaxial tension or compression along $x_{3}: \bar{\sigma}_{11}=\bar{\sigma}_{22}=0$. 
pressure $\bar{p}$ can be readily calculated provided the overall deviatoric stress $\bar{\sigma}^{\prime}$ is known from the VPSC scheme. The overall and the local Cauchy stresses are subsequently obtained as:

$$
\begin{aligned}
\bar{\sigma}_{i j} & =\bar{\sigma}_{i j}^{\prime}+\bar{p} \delta_{i j} \\
\sigma_{i j} & =\sigma_{i j}^{\prime}+(\bar{p}+\tilde{p}) \delta_{i j} .
\end{aligned}
$$

The local stress states given by equation (29b) (obtained along a certain loading path) are the stresses in each grain for the polycrystal under load. In order to calculate the residual stresses it is necessary to unload the polycrystal, bringing to zero the overall stress. The elastic unloading is calculated here by means of an elastic self-consistent model. Since elasticity represents a truly linear relation between stress and (elastic) strain, the results of the linearized viscoplastic model of the previous section apply exactly to elasticity. In particular, equations (20)-(23) and (28) adopt the same algebraic form in elasticity, with viscoplastic compliance, stiffness, and Eshelby tensors being replaced by the elastic counterparts, and with the strain rate and deviatoric stress being replaced by the elastic strain and the Cauchy stress. (See Hill [5], for details on the elastic self-consistent polycrystal formulation). As a consequence, the localization equation now applies to the six independent components of the Cauchy stress and reads:

$$
\boldsymbol{\sigma}=\boldsymbol{B}^{c(\mathrm{el})}: \overline{\boldsymbol{\sigma}} \text {. }
$$

When unloading the aggregate, it is necessary to transfer from the viscoplastic to the elastic regime. The transition has to be such that the local stresses and the macroscopic applied stress calculated with the VPSC scheme become the initial loaded state from which an elastic unloading is performed using the self-consistent elastic formulation. It is obvious, from a comparison of the localization equations (27) and (30), that the same overall stress $\bar{\sigma}$ will induce different internal stresses under the viscoplastic and the elastic regimes. The way to preserve the same internal stress in the grains is to introduce a transformation strain in each grain, that preserves the local stresses when the transition from the viscoplastic to the elastic regime is performed. Such a transformation strain can be introduced by writing the localization equation (27) in an alternative form, as:

$$
\boldsymbol{\sigma}=\boldsymbol{B}^{c(\mathrm{el})}: \overline{\boldsymbol{\sigma}}+\left(\boldsymbol{B}^{c(\mathrm{xvp})}-\boldsymbol{B}^{c(\mathrm{el})}\right): \overline{\boldsymbol{\sigma}} .
$$

The first term on the right-hand side is the stress localization in the grain induced by loading elastically with the overall stress $\bar{\sigma}$ (see equation (30)), while the second term may be interpreted as the fictitious transformation that keeps the stress in the grain invariant. Unloading elastically amounts to bringing the overall stress to zero in the first term, which gives a residual stress $\boldsymbol{\sigma}^{\text {res }}$ in each grain as:

$$
\boldsymbol{\sigma}^{\mathrm{res}}=\left(\boldsymbol{B}^{c(\mathrm{xvp})}-\boldsymbol{B}^{c(\mathrm{el})}\right): \overline{\boldsymbol{\sigma}} \text {. }
$$

For future comparison with experiments and discussion, it is useful to define the following elastic strains in the grain. The residual strain, associated with the residual stress after unloading, is:

$$
\epsilon^{\mathrm{res}}=M^{c(\mathrm{el})}: \boldsymbol{\sigma}^{\mathrm{res}} \text {. }
$$

The elastic strain under load is:

$$
\epsilon^{\text {load }}=M^{c(\mathrm{el})}: \boldsymbol{\sigma}
$$

while the elastic intergranular strain under load is defined as the difference between the elastic strain in the grain and the overall elastic strain:

$$
\epsilon^{\mathrm{int}}=\boldsymbol{M}^{c(\mathrm{el})}: \boldsymbol{\sigma}-\boldsymbol{M}^{(\mathrm{el})}: \overline{\boldsymbol{\sigma}} \text {. }
$$




\section{Results and discussion}

\subsection{Incoloy-800 - $12 \%$ tension}

The model described in the previous sections has been applied to the prediction of residual strains and stresses in Incoloy-800, an austenitic alloy with weight per cent composition $\mathrm{Fe}_{45} \mathrm{Ni}_{33} \mathrm{Cr}_{22}$, which is used in heat exchangers for its resistance to water corrosion. The works of Holden et al [16] and Leitch and Tomé [17] analyse the distribution of residual strains in Incoloy-800 tubes after plastic bending. Recently, Holden et al [18] have used neutron diffraction to measure crystallographic lattice spacings in samples cut from rolled Incoloy-800 along the rolling direction of the sheet, and subsequently deformed in tension. Plastic deformation was accumulated by cyclic loading and unloading, while simultaneously measuring the evolution of lattice spacings along RD.

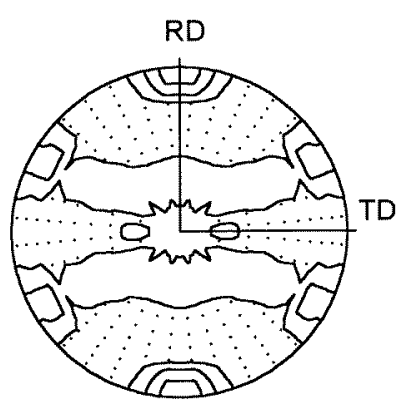

(a) initial texture

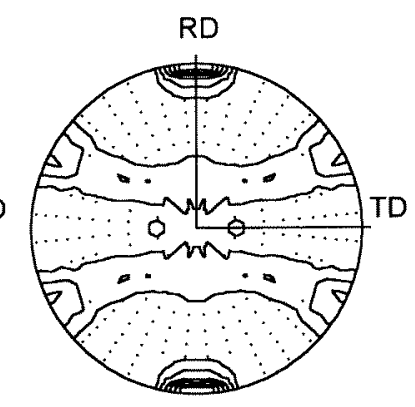

(b) $12 \%$ tension

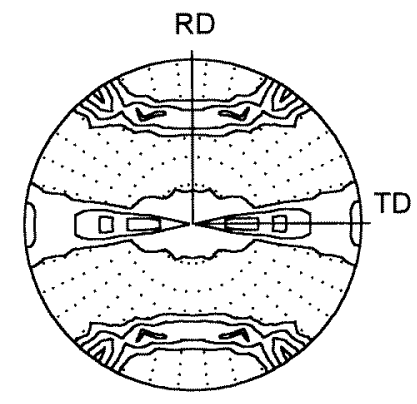

(c) $50 \%$ compression

Figure 1. (111) pole figure of:(a) rolled Incoloy-800 texture measured by Holden et al [18]; (b) predicted texture after $12 \%$ tensile deformation along the $\mathrm{RD}$; $(c)$ predicted texture after $50 \%$ compressive deformation along the RD.

In order to compare the present model predictions with these measurements, a uniaxial tension test was simulated using an initial texture of 1356 discrete orientations with weights, generated directly from the orientation distribution function measured by Holden et al [18]. The (111) pole figure is shown in figure $1(a)$. This texture exhibits a dominant $\{111\}$ and a secondary $\{100\}$ component along the RD, i.e. a $21.2 \%$ and a $7.2 \%$ volume fraction of grains have their $\{111\}$ and $\{100\}$ normals within $7.5^{\circ}$ of RD, respectively. An initial spherical grain shape and a value of $n=19$ for the reciprocal of the rate sensitivity were adopted. The value used for the initial CRSS on the $\{111\}\langle 110\rangle$ slip system is $\tau_{0}=85 \mathrm{MPa}$ and the coefficient of microhardening (assumed isotropic) is $h=330 \mathrm{MPa}$. The increment of the CRSS of the slip system $(s)$ is given by:

$$
\Delta \tau_{0}^{s}=h \times \sum_{s^{\prime}} \Delta \gamma^{s^{\prime}} .
$$

The latter parameters were adjusted to match the loading curve measured by Holden et al [18], shown in figure 2. The dotted line in figure 2 indicates the method used in VPSC to simulate the deformation process, i.e. a uniform stress state which induces a certain strain rate is assumed to be applied to the polycrystal during a time interval. After each time interval (fixed so that the strain increment is 0.02) the orientations and the critical stresses are updated and, therefore, the next incremental step requires a higher applied stress.

Uniaxial tension along the RD was simulated up to $12 \%$ strain (figure $1(b)$ ), starting from the initial rolling texture (figure 1(a)), and imposing zero lateral stresses on the sample. 


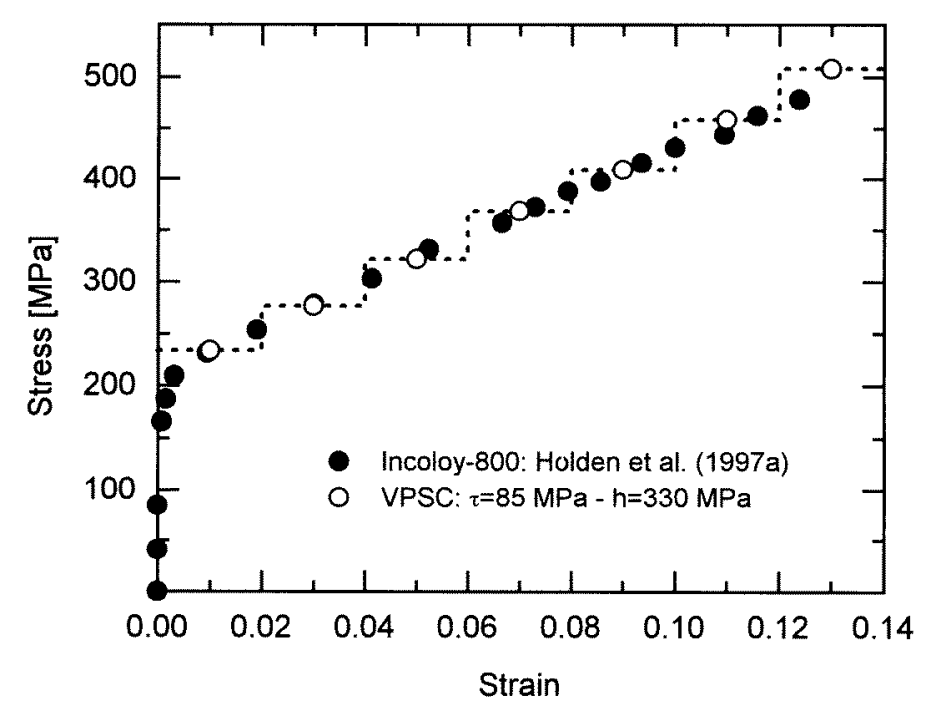

Figure 2. Loading curve along the RD for Incoloy-800 measured by Holden et al [18] (solid symbols) and predicted with the VPSC model (dotted line and open symbols) using $\tau_{0}=85 \mathrm{MPa}$ and $h=330 \mathrm{MPa}$.

In addition, deformation up to $50 \%$ strain in compression was simulated to investigate the effect of texture development upon the residual stresses (see section 3.2). It can be seen in figure 1 that, while the tensile deformation reinforces the $\{111\}$ component along the RD, compression reduces this component.

The single-crystal elastic constants used for the elastic unloading were also taken from Holden et al [18]: $C_{11}=218 \mathrm{GPa}, C_{12}=141 \mathrm{GPa}, C_{44}=115 \mathrm{GPa}$. These values show that Incoloy-800 has a high elastic anisotropy: the directional Young moduli along $\langle 111\rangle$ and $\langle 100\rangle$ are $E_{\langle 111\rangle}=218 \mathrm{GPa}$ and $E_{\langle 100\rangle}=107 \mathrm{GPa}$, respectively. The plastic anisotropy of an FCC single crystal such as Incoloy-800 is also marked: the Taylor factors for a single crystal under uniaxial tension are 3.67 and 2.45 , along the $\langle 111\rangle$ and $\langle 100\rangle$ directions, respectively.

The extended VPSC model can be used to calculate the elastic strain of the grains that have a given crystallographic direction along a particular direction in the space. In the present case, $\mathrm{RD}$ is an important direction. The comparison with the measurements of Holden et al [18] requires the computation of the average longitudinal component of the elastic intergranular strain under load and the residual strain (referred to, for brevity, in what follows as 'elastic strains') for grains having their $\{111\}$ and $\{100\}$ normals along RD.

Table 1 shows such a comparison. From a qualitative point of view, both under load and after unloading, the signs of the predicted elastic strains match the experiments. But, on the other hand, the model overestimates the strains by factors of 1.5-3, depending on the component. The relative values of the predicted elastic strains are consistent with the main components of the texture, i.e. the strains on the secondary $\{100\}$ component are larger than and opposite in sign to those on the predominant $\{111\}$ grains, in order to compensate for the smaller fraction of $\{100\}$ grains.

An interesting and apparently contradictory result is that, for both the $\langle 111\rangle$ and the $\langle 100\rangle$ directions, the signs of the intergranular strains under load are the same as those of the residual strains (negative and positive respectively). This can be explained in terms of 
Table 1. Residual and intergranular elastic strains (equations (33) and (35)) for grains with $\{111\}$ and $\{100\}$ planes having their normal aligned with the RD of an Incoloy-800 plate. Measured by Holden et al [18] and calculated with the VPSC model, after 12\% tension along the RD. Units of $10^{-4}$.

\begin{tabular}{llllll}
\hline & \multicolumn{2}{c}{111} & & \multicolumn{2}{c}{100} \\
\cline { 2 - 3 } \cline { 5 - 6 } & $\begin{array}{l}\text { Intergranular } \\
\text { under load }\end{array}$ & Residual & & $\begin{array}{l}\text { Intergranular } \\
\text { under load }\end{array}$ & Residual \\
\hline Predicted & -4.1 & -1.4 & & 21.2 & 14.9 \\
Measured & -3.5 & -0.8 & & 7.8 & 7.9 \\
\hline
\end{tabular}

the relative elastic and plastic anisotropy. Figure 3 shows schematically the local response of typical $\{111\}$ and $\{100\}$ grains together with the overall response of the polycrystal (label PX) assuming that the directional Young modulus and the Taylor factor of the $\{111\}$ grains are higher than those of the $\{100\}$ grains. For the $\{111\}$ grain, although the yield stress is higher than for $\{100\}$, it takes less elastic strain than the average to reach that point because of the high elastic stiffness. Therefore, the elastic intergranular strain under load (equation (35)) is negative. During unloading, the elastic slope of the $\{111\}$ grain is higher than the average slope and eventually the local elastic strain changes sign before the overall stress is completely removed, giving a final negative residual strain (equation (33)). An appropriate treatment of the local elastic and plastic anisotropy, as provided by the self-consistent formulation, has been essential to capture this effect.

The model also allows one to study the variation of the elastic strains as a function of the sample direction in which they are measured. An example of this is shown in figure 4 for

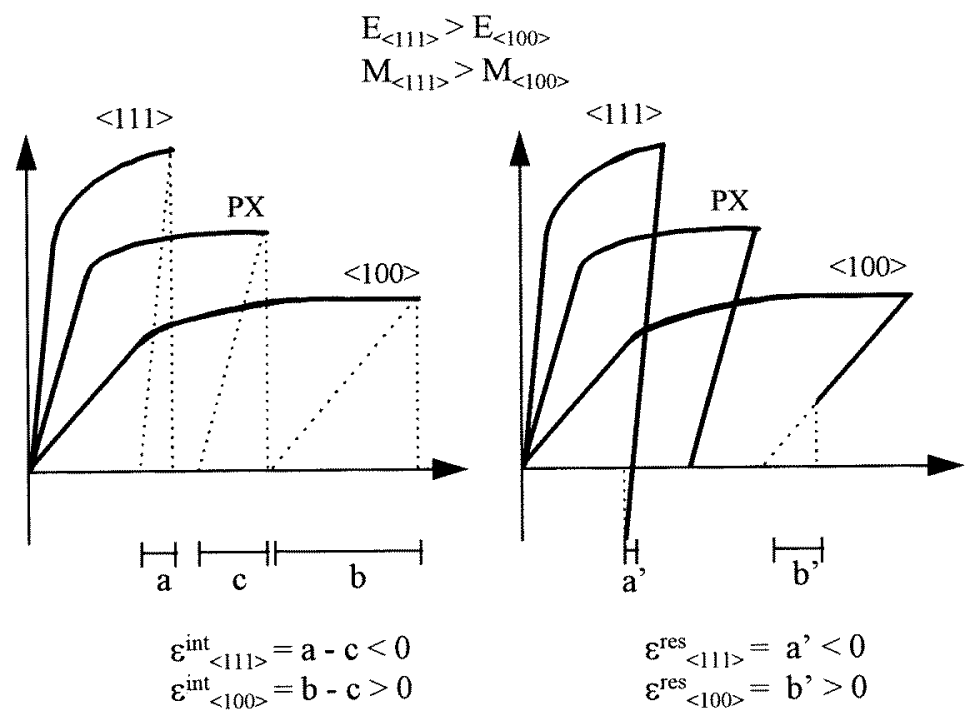

Figure 3. Schematic representation of the local and overall (PX) response of a textured polycrystal with a $\{111\}$ and a $\{100\}$ texture component along the loading direction. The directional Young modulus $\left(E_{\langle h k l\rangle}\right.$ and Taylor factor $\left(M_{\langle h k l\rangle}\right)$ of the $\{111\}$ grains are higher than those of the $\{100\}$ grains. 

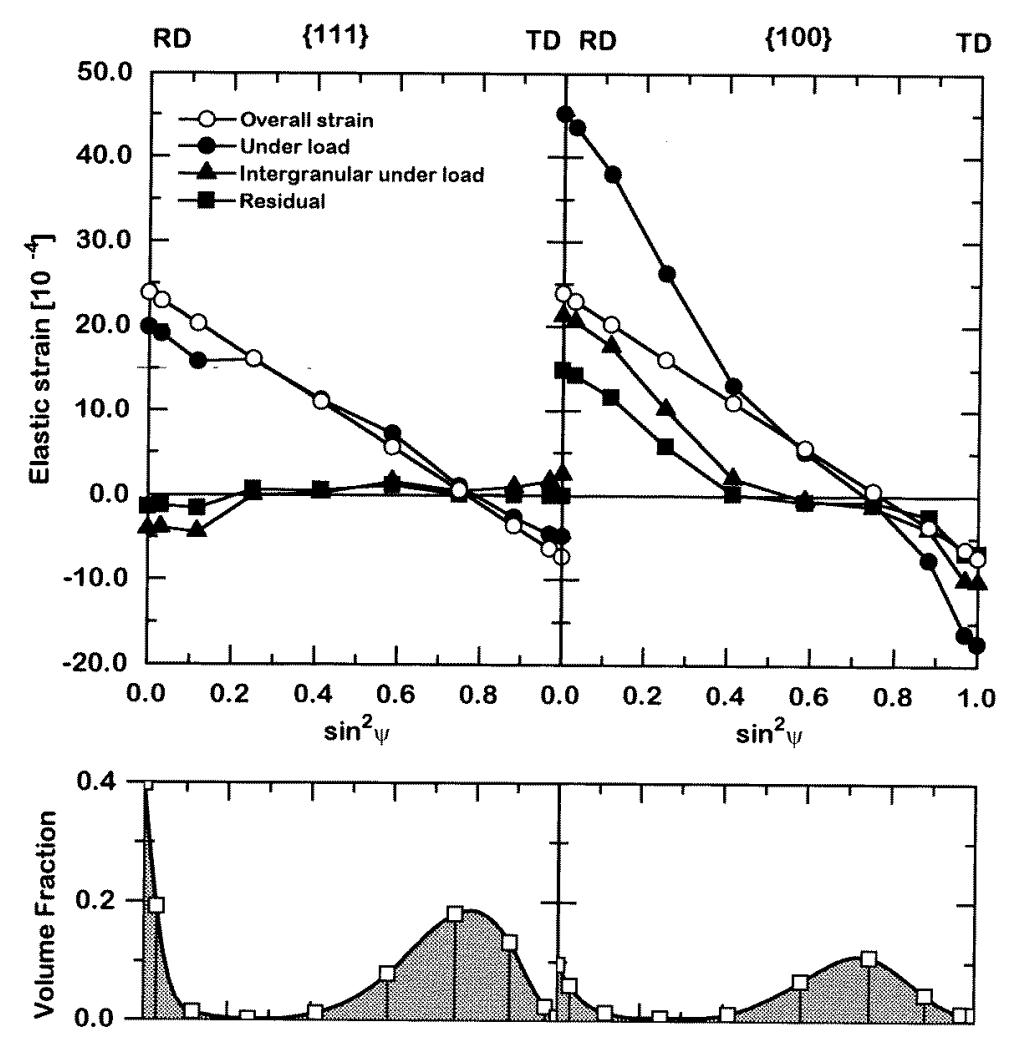

Figure 4. Calculated elastic strains after $12 \%$ tension in textured Incoloy- 800 for grains with $\{111\}$ and $\{100\}$ normals at an angle $\psi$ from the RD towards the TD. Shaded regions are proportional to the volume fraction of grains in diffracting conditions.

grains with their $\{111\}$ and $\{100\}$ normals at an angle $\psi$ measured from the RD towards the TD. In addition to the strain components along those directions of $\epsilon^{\text {res }}, \epsilon^{\text {load }}, \epsilon^{\text {int }}$ (equations (33)-(35)) and of the macroscopic strain, the shaded regions at the bottom of the figure represent, for each direction, the volume fraction of material in diffracting condition. While the overall strain, which is by definition homogeneous, shows the classical linear behaviour in this $\sin ^{2} \psi$ plot, the other elastic strains depart from such behaviour. This departure, which is particularly dramatic for the residual strains of the $\{100\}$ grains, is associated with the local heterogeneity of elastic and plastic deformation and is discussed in detail by Holden et al [19].

\subsection{Effect of texture development}

One of the main advantages of the present model is that it can take account of the development of texture and of internal states simultaneously. However, in the previous example of $12 \%$ tension texture development does not play an important role, since it is a relatively low deformation and the major texture components are reinforced under such a strain path. Therefore, calculations for $50 \%$ compressive strain along the RD will be presented in what follows, because they have associated substantial grain reorientations and, as a consequence, texture variations. In these simulations the grain's reorientation can be 
Table 2. Predicted residual strains (equation (33)) for grains with $\{111\}$ and $\{100\}$ planes having their normal aligned with RD and TD of an Incoloy- 800 plate, after $12 \%$ tension and $50 \%$ compression along the RD, with and without texture evolution. In parentheses: volume fraction of grains that fulfil the diffraction condition. Units of $10^{-4}$.

\begin{tabular}{|c|c|c|c|c|c|c|c|c|}
\hline & \multicolumn{4}{|c|}{$12 \%$ tension } & \multicolumn{4}{|c|}{$50 \%$ compression } \\
\hline & \multicolumn{2}{|c|}{111} & \multicolumn{2}{|c|}{100} & \multicolumn{2}{|c|}{111} & \multicolumn{2}{|c|}{100} \\
\hline & $\mathrm{RD}$ & $\mathrm{TD}$ & $\mathrm{RD}$ & $\mathrm{TD}$ & $\mathrm{RD}$ & $\mathrm{TD}$ & $\mathrm{RD}$ & $\mathrm{TD}$ \\
\hline $\begin{array}{l}\text { Without texture } \\
\text { evolution }\end{array}$ & $\begin{array}{l}-1.3 \\
(21.2 \%)\end{array}$ & $\begin{array}{c}0.3 \\
(0.8 \%)\end{array}$ & $\begin{array}{l}14.2 \\
(7.2 \%)\end{array}$ & $\begin{array}{l}-5.2 \\
(0.8 \%)\end{array}$ & $\begin{array}{l}2.6 \\
(21.2 \%)\end{array}$ & $\begin{array}{c}0.5 \\
(0.8 \%)\end{array}$ & $\begin{array}{l}-28.1 \\
(7.2 \%)\end{array}$ & $\begin{array}{l}14.2 \\
(1.5 \%)\end{array}$ \\
\hline $\begin{array}{l}\text { With texture } \\
\text { evolution }\end{array}$ & $\begin{array}{l}-1.4 \\
(39.8 \%)\end{array}$ & $\begin{array}{l}-0.1 \\
(0.5 \%)\end{array}$ & $\begin{array}{l}14.9 \\
(9.5 \%)\end{array}$ & $\begin{array}{l}-6.7 \\
(0.5 \%)\end{array}$ & $\begin{array}{l}1.6 \\
(0.8 \%)\end{array}$ & $\begin{array}{l}-3.8 \\
(14.7 \%)\end{array}$ & $\begin{array}{l}-22.9 \\
(1.0 \%)\end{array}$ & $\begin{array}{c}1.0 \\
(10.2 \%)\end{array}$ \\
\hline
\end{tabular}

deactivated (i.e. 'freezing' the initial texture) in order to study the effect of texture evolution. Table 2 shows the intergranular strains under load and the residual strains for grains having their $\{111\}$ and $\{100\}$ normals along RD of the Incoloy-800 plate, for $12 \%$ tension and $50 \%$ compression, with and without texture evolution. In addition, in parentheses, the table shows the volume fraction of grains in diffracting condition. In the $12 \%$ tension case, the texture development essentially consists of a reinforcement of the $\{111\}$ and $\{100\}$ components along RD (see figure $1(b)$ ). The predicted residual strains along RD with and without texture evolution show only minor differences. On the other hand, there is a drastic change of the texture after 50\% compression (see figure 1(c)), namely: the $\langle 111\rangle$ and $\langle 100\rangle$ directions are predominantly aligned with TD instead of RD. Consequently, the predicted elastic strains along TD present important differences when calculated with and without texture evolution.

\subsection{Comparison with other models}

The inquisitive reader must be wondering how the strains predicted using this extended VPSC model compare with the predictions of a better established EPSC model, such as that of Hill [5]. As we argue in the introduction, the VPSC method is a well established method for the description of texture evolution and large-deformation response, and it is the evolution of plastic properties (not elasticity) that controls such a regime. However, does one expect the EPSC model to converge to the VPSC at large strains, provided that one does not take into account grain reorientation? Before answering this question we have to bring into consideration other features of these models. The VPSC formulation is based on an interaction between inclusion and HEM (equation (21)) described by the tangent compliance. This interaction is $n$ times more compliant than that described by the secant compliance (equation (22)), and allows for larger strain deviations in the grains from the average. In other words, a secant formulation gives results which are closer to an upper bound, a feature which is connected to Hutchinson's viscoplastic self-consistent model [14]. The latter model is based on an incremental formulation, where a linear relation is assumed between strainrate increments and stress increments, and the results tend to coincide with those for an upper-bound (Taylor) model as the reciprocal of the rate sensitivity $n$ increases. Lebensohn and Tomé [12] discuss such a response, and argue that the incremental formulation is equivalent to adopting a secant stiffness in a non-incremental formulation. More recently, Molinari et al [6] arrive at the same conclusion via a more formal analysis and critical 
comparison of several elasto-plastic, visco-elastic and viscoplastic models.

Now, as far as the EPSC model of Hill [5] and Hutchinson [20] is concerned, this is an incremental formulation where stress increments are linearly related to strain increments. As a consequence, this is also characterized by a secant stiffness and a rather rigid interaction [6]. We are not implying here that tangent is more appropriate than secant for describing the heterogeneity in the response of aggregates, and the truth most probably lies in between the two cases. However, independently of this issue, if we wish to compare the residual stresses predicted with our extended VPSC model and those predicted with the EPSC formulation of Hill, we need to use the second version (equation (22)) of the VPSC model.

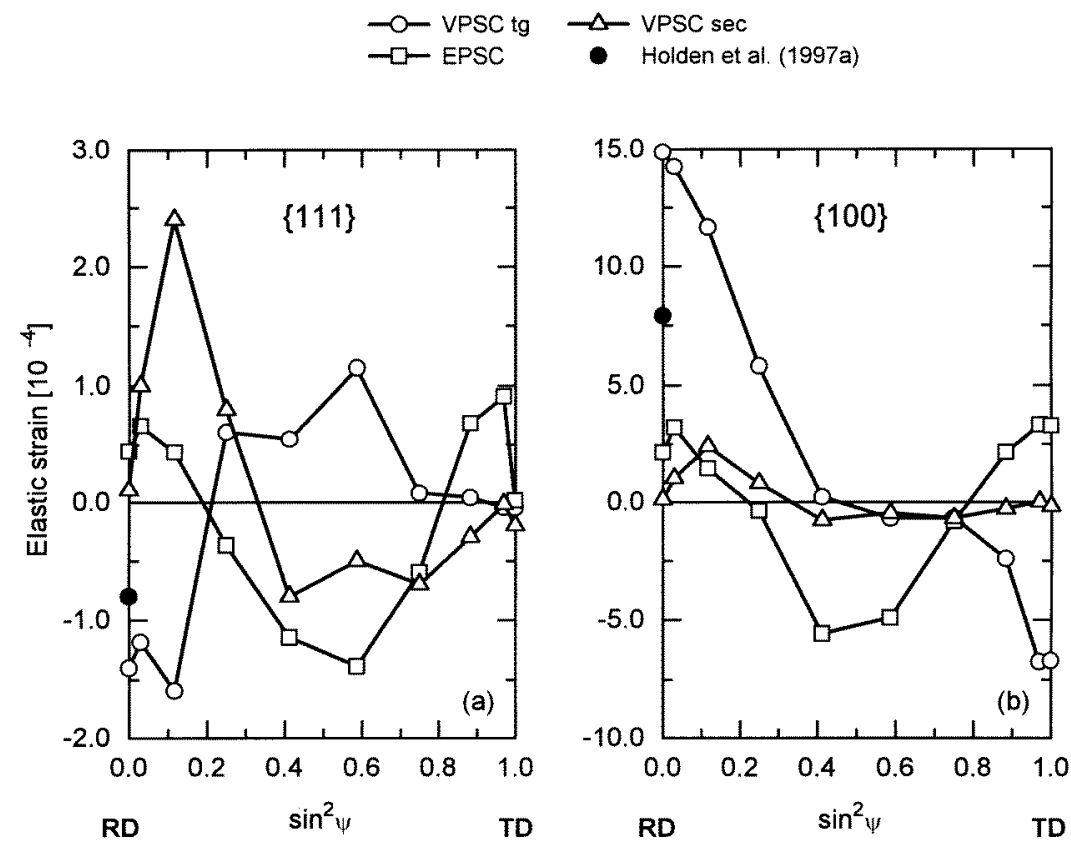

Figure 5. Elastic residual strain after $12 \%$ tension along the RD of an Incoloy- 800 sheet, for grains with $\{111\}$ and $\{100\}$ normals at an angle $\psi$ from the RD towards the TD, calculated with EPSC, secant VPSC and tangent VPSC models. Solid symbols correspond to values measured by Holden et al [18].

Figure 5 shows predictions in a $\sin ^{2} \psi$ plot for the textured Incoloy- 800 plate after $12 \%$ tension along RD, calculated with the tangent VPSC, the secant VPSC and the EPSC models (see Turner and Tomé [21] for a description of the implementation of the HillHutchinson EPSC model). Observe, on the hand, that the intergranular strains induced by the mechanical loading introduce a clear deviation from the linear dependence upon $\sin ^{2} \psi$ predicted by homogeneous continuum deformation theory. We verified that the effect of texture evolution is not important after 12\% tension, and the differences between the EPSC and the two VPSC models are mainly due to the different constitutive behaviours assumed by each model. It is clear from the residual strains plotted in figure 5 for $\{111\}$ and $\{100\}$ orientations that, despite some quantitative differences, the secant form of the extended VPSC model and the EPSC model predict similar profiles of residual stresses. In particular, both approximations give very small residual strains in $\{111\}$ and $\{100\}$ planes along RD, unlike the measurements of Holden et al [18], which indicate the presence of non-negligible 
strains in both cases.

The tangent VPSC model, on the other hand, predicts results which are not only quantitatively different from those for the EPSC and the secant VPSC models, as is evident from figure 5. Specifically, the tangent VPSC predicts the compressive strain in the $\{111\}$ planes aligned with RD, and the tensile strain in the $\{100\}$ planes, in agreement with result of Holden et al [18].

\section{Conclusions}

We present an extended VPSC model for the calculation of residual stresses associated with cold working as an alternative to performing an elasto-plastic simulation of the aggregate deformation. This approach, which is an extension of well established plastic deformation models, allows us to keep track of the evolution of the texture and the local Cauchy stress in each grain of a polycrystal, simultaneously. Our approach is based on a selfconsistent solution of the incompressible inclusion problem, where the local deviatoric and pressure components of the stress follow from solving equilibrium and the condition of incompressibility. We propose a way of transferring from the elasto-plastic regime to an elastic regime that leaves the local and overall stress tensors invariant, followed by an elastic self-consistent unloading that gives the intergranular residual states in the grains. The model is used to predict residual strains in rolled Incoloy-800 deformed in tension and compression, and a comparison is performed with available experimental estimates. This comparison indicates that the results of this approach are consistent with the experimental evidence. The results are also compared with the predictions of a stiffer VPSC model and a classical EPSC model.

While it is not the intention of this work to validate the model based on the limited comparison with experimental evidence, it is reassuring to observe that the two are consistent. A critical validation of the model would require the exploration of several alternatives, which is not the aim of this paper. A possible improvement would be to use an elasto-plastic unloading instead of a pure elastic springback in order to calculate the residual stresses. According to our experience, the amount of plastic deformation induced by intergranular interactions during the unloading stage is negligible for cubic and hexagonal aggregates [21]. Any premature Bauschinger effect observed before the stress crosses the horizontal axis is more probably due to intragranular dislocations mechanisms, such as recovery of dislocations of opposite sign, a feature which is not taken into account by any of the polycrystal models discussed above.

Here, we assume isotropic crystallographic hardening in order simplify the analysis, but we have verified that hardening plays an important role in determining the final residual strains after unloading. Latent and self-hardening schemes will have to be explored when making a critical comparison of models. Furthermore, validation of the model will require comparison with measurements of residual strains performed for different crystallographic planes, in different directions, at different amounts of deformation and for different deformation modes. Such a comparison was recently made by Tomé et al [22] for another Ni based alloy of cubic structure, Monel-400. In particular, the latter authors compare the predictions of the three models discussed above with experimentally measured residual strains. They conclude that none of the models has a clear advantage in reproducing all the experimental results, at least within the strain range for which data are available $(\epsilon<8 \%)$.

Whereas in this work we use the incompressibility condition to calculate the hydrostatic stress component in each grain before transferring to the elastic regime, other approaches are 
feasible. For example, the local pressure may be given by an elasto-plastic self-consistent calculation, for an aggregate loaded with the overall stress $\bar{\sigma}$. It is interesting to notice that the local pressure given by such an approach is uniform for a cubic aggregate loaded elastically. It is not uniform, however, for non-cubic aggregates or for cubic aggregates deforming elasto-plastically. Independently of the procedure used to evaluate the local pressure, the transition between the viscoplastic and the elastic regime still requires the use of a fictitious transformation strain (equation (31)) in order to keep the local and overall stress tensors invariant.

In conclusion, we should like to state that the extended VPSC model seems to be a valid option for calculating residual strains associated with cold-working operations. While it can be used to address large strains and texture development, the method is not concerned with stress spins. Instead, reorientation and local stress updates take place incrementally, via relatively large steps of about $2 \%$ deformation. This represents an advantage over elastoplastic models, which require much smaller strain increments (of about $0.01 \%$ ) and longer execution time. The disadvantage is that, since the model disregards the elastic component during loading, it is not applicable to small-strain regimes $(\epsilon<2 \%)$ where the elastic and the plastic components are comparable.

\section{Appendix A. Separation of deviatoric and hydrostatic components}

When dealing with incompressible media, it is convenient to explicitly decompose stress and strain rate into deviatoric and hydrostatic components and to confine them to different subspaces, which may be uncoupled for certain mechanical regimes. There are different ways of achieving such a decomposition and the formulation described in section 2 of this work is independent of the particular convention. In particular, for our calculations we express second- and fourth-order tensorial quantities in an orthonormal basis of second-order symmetric tensors $\left\{\boldsymbol{b}^{(\lambda)}\right\}$, defined as:

$$
\begin{array}{lcc}
b^{(1)}=\frac{1}{\sqrt{6}}\left(\begin{array}{rrr}
-1 & 0 & 0 \\
0 & -1 & 0 \\
0 & 0 & 2
\end{array}\right) & b^{(2)}=\frac{1}{\sqrt{2}}\left(\begin{array}{rrr}
-1 & 0 & 0 \\
0 & 1 & 0 \\
0 & 0 & 0
\end{array}\right) \quad b^{(3)}=\frac{1}{\sqrt{2}}\left(\begin{array}{lll}
0 & 0 & 0 \\
0 & 0 & 1 \\
0 & 1 & 0
\end{array}\right) \\
b^{(4)}=\frac{1}{\sqrt{2}}\left(\begin{array}{lll}
0 & 0 & 1 \\
0 & 0 & 0 \\
1 & 0 & 0
\end{array}\right) & b^{(5)}=\frac{1}{\sqrt{2}}\left(\begin{array}{lll}
0 & 1 & 0 \\
1 & 0 & 0 \\
0 & 0 & 0
\end{array}\right) \quad b^{(6)}=\frac{1}{\sqrt{3}}\left(\begin{array}{lll}
1 & 0 & 0 \\
0 & 1 & 0 \\
0 & 0 & 1
\end{array}\right)
\end{array}
$$

The components of this basis have the property:

$$
b_{i j}^{\lambda} b_{i j}^{\lambda \prime}=\delta_{\lambda \lambda^{\prime}}
$$

and provide a unique 'vector' and 'matrix' representation of second- and fourth-order symmetric tensors, respectively. In the particular case of the stress tensor:

$$
\sigma_{i j}=\sigma_{\lambda} b_{i j}^{\lambda} \quad(\text { sum on } \lambda)
$$

where:

$$
\sigma_{\lambda}=\sigma_{i j} b_{i j}^{\lambda} \quad(\lambda=1, \ldots, 6) .
$$

The orthonormality of the basis guarantees that the six-dimensional strain-rate and stress vectors are work conjugate, i.e.

$$
\dot{\epsilon}_{\lambda} \sigma_{\lambda}=\dot{\epsilon}_{i j} \sigma_{i j} \text {. }
$$


The explicit form of the components $\sigma_{\lambda}$ is:

$$
\left(\frac{2 \sigma_{33}-\sigma_{11}-\sigma_{22}}{\sqrt{6}}, \frac{\sigma_{22}-\sigma_{11}}{\sqrt{2}}, \sqrt{2} \sigma_{23}, \sqrt{2} \sigma_{13}, \sqrt{2} \sigma_{12}, \frac{\sigma_{11}+\sigma_{22}+\sigma_{33}}{\sqrt{3}}\right) .
$$

and similarly for the components $\epsilon_{\lambda}$. These 'vector' components coincide with those used by Lebensohn and Tomé [12]. It is clear that in this representation the first five components are deviatoric and the sixth is proportional to the hydrostatic component of the tensor. The deviatoric stress and the incompressible strain rate are given by the first five components as:

$$
\begin{array}{ll}
\sigma_{i j}^{\prime}=\sigma_{\lambda} b_{i j}^{\lambda} & (\lambda=1, \ldots, 5) \\
\dot{\epsilon}_{i j}=\dot{\epsilon}_{\lambda} b_{i j}^{\lambda} & (\lambda=1, \ldots, 5) .
\end{array}
$$

The viscoplastic constitutive laws given by equations (2) and (5) are expressed in the cartesian space, where they have the disadvantage of being non-invertible because the compliance tensor of an incompressible medium is singular in the cartesian space. In what follows, we show how they can be inverted when represented in a five-dimensional deviatoric subspace. The procedure will be applied to the more general tangent form of the constitutive equation, although the same considerations apply to the secant form. Equation $(2 b)$ is expressed in terms of the cartesian components as:

$$
\dot{\epsilon}_{i j}=M_{i j k l}^{(\mathrm{tan})} \sigma_{k l}^{\prime}+\dot{\epsilon}_{i j}^{0} .
$$

Contracting both members of this equation with $\boldsymbol{b}^{\lambda}$ and using (A7), this equation becomes:

$$
b_{i j}^{\lambda} \dot{\epsilon}_{i j}=b_{i j}^{\lambda} M_{i j k l}^{(\mathrm{tan})} b_{k l}^{\lambda^{\prime}} \sigma_{\lambda^{\prime}}+b_{i j}^{\lambda} \dot{\epsilon}_{i j}^{0} \quad\left(\lambda, \lambda^{\prime}=1, \ldots, 5\right)
$$

that is:

$$
\dot{\epsilon}_{\lambda}=M_{\lambda \lambda^{\prime}}^{(\tan )} \sigma_{\lambda^{\prime}}+\dot{\epsilon}_{\lambda}^{0} \quad\left(\lambda, \lambda^{\prime}=1, \ldots, 5\right)
$$

where $\left(M^{(\tan )}\right)$ is a $5 \times 5$ matrix defined by the components of the tensor in the basis $b^{\lambda}$ as:

$$
M_{\lambda \lambda^{\prime}}^{(\mathrm{tan})}=M_{i j k l}^{(\mathrm{tan})} b_{i j}^{\lambda} b_{k l}^{\lambda^{\prime}} \quad\left(\lambda, \lambda^{\prime}=1, \ldots, 5\right) .
$$

Equation (A11) can be inverted in the five-dimensional subspace, giving:

$$
\sigma_{\lambda}=L_{\lambda \lambda^{\prime}}^{(\tan )} \dot{\epsilon}_{\lambda^{\prime}}+\sigma_{\lambda}^{0} \quad\left(\lambda, \lambda^{\prime}=1, \ldots, 5\right)
$$

where $\left(L^{(\tan )}\right)=\left(M^{(\tan )}\right)^{-1}$ is a well defined $5 \times 5$ matrix that determines a linear relation between the deviatoric stress components and the deviatoric strain rate components and $\sigma_{\lambda}^{0}$ is a back-extrapolated stress. Equation (A13) can now be expressed in terms of the cartesian tensor components:

$$
\sigma_{i j}^{\prime}=L_{i j k l}^{(\mathrm{tan})} \dot{\epsilon}_{k l}-\sigma_{k l}^{\prime 0}
$$

where

$$
L_{i j k l}^{(\tan )}=L_{\lambda \lambda^{\prime}}^{(\tan )} b_{i j}^{\lambda} b_{k l}^{\lambda^{\prime}} \quad\left(\lambda, \lambda^{\prime}=1, \ldots, 5\right) .
$$




\section{Appendix B. Solution of the viscoplastic inclusion in an incompressible medium}

The solution of the viscoplastic inclusion embedded in an incompressible medium was derived by Molinari et al [11]. Here we reproduce their formulation in more detail, in order to derive explicit integrals for the evaluation of local strain-rate and pressure deviations in anisotropic media. The goal is to solve the system (15) of four differential equations with four unknowns: the velocity field deviation $\tilde{\dot{u}}$ and the pressure deviation $\tilde{p}$. The Green functions for the velocity field, $G_{i j}(\overline{\boldsymbol{r}})$, and for the pressure, $H_{i}(\overline{\boldsymbol{r}})$, are the solutions of the following set of equations:

$L_{i j k l}^{(\mathrm{tan})} G_{k m, l j}\left(\overline{\boldsymbol{r}}-\overline{\boldsymbol{r}}^{\prime}\right)+H_{m, i}\left(\overline{\boldsymbol{r}}-\overline{\boldsymbol{r}}^{\prime}\right)+\delta_{i m} \delta\left(\overline{\boldsymbol{r}}-\overline{\boldsymbol{r}}^{\prime}\right)=0 \quad G_{k m, k}\left(\overline{\boldsymbol{r}}-\overline{\boldsymbol{r}}^{\prime}\right)=0$.

The Fourier transforms of the Green functions are defined by:

$$
\begin{aligned}
& \hat{G}_{i j}(\overline{\boldsymbol{k}})=\int_{\mathbb{R}^{3}} G_{i j}(\overline{\boldsymbol{r}}) \exp (\mathrm{i} \overline{\boldsymbol{k}} \cdot \overline{\boldsymbol{r}}) \mathrm{d} \overline{\boldsymbol{r}} \\
& \hat{H}_{i}(\overline{\boldsymbol{k}})=\int_{\mathbb{R}^{3}} H_{i}(\overline{\boldsymbol{r}}) \exp (\mathrm{i} \overline{\boldsymbol{k}} \cdot \overline{\boldsymbol{r}}) \mathrm{d} \overline{\boldsymbol{r}} .
\end{aligned}
$$

Applying the Fourier transform to (B1) converts the set of differential equations into an algebraic linear system:

$\alpha_{l} \alpha_{j} L_{i j k l}^{(\tan )}\left(k^{2} \hat{G}_{k m}(\overline{\boldsymbol{k}})\right)+\alpha_{i}\left(i k \hat{H}_{m}(\overline{\boldsymbol{k}})\right)=\delta_{i m} \quad \alpha_{k}\left(k^{2} \hat{\boldsymbol{G}}_{k m}(\overline{\boldsymbol{k}})\right)=0$

where $\overline{\boldsymbol{\alpha}}$ is the unit vector along the direction of $\overline{\boldsymbol{k}}$ (a vector of the transformed space), i.e. $\overline{\boldsymbol{k}}=k \overline{\boldsymbol{\alpha}}$. Calling $A_{i k}=\alpha_{l} \alpha_{j} L_{i j k l}^{\text {(tan) }}$, equation (B3) can be re-written as:

$$
\left(\begin{array}{cccc}
A_{11} & A_{12} & A_{13} & \alpha_{1} \\
A_{21} & A_{22} & A_{23} & \alpha_{2} \\
A_{31} & A_{32} & A_{33} & \alpha_{3} \\
\alpha_{1} & \alpha_{2} & \alpha_{3} & 0
\end{array}\right) \times\left(\begin{array}{ccc}
k^{2} \hat{G}_{11} & k^{2} \hat{G}_{12} & k^{2} \hat{G}_{13} \\
k^{2} \hat{G}_{21} & k^{2} \hat{G}_{22} & k^{2} \hat{G}_{23} \\
k^{2} \hat{G}_{31} & k^{2} \hat{G}_{32} & k^{2} \hat{G}_{33} \\
i k \hat{H}_{1} & i k \hat{H}_{2} & i k \hat{H}_{3}
\end{array}\right)=\left(\begin{array}{ccc}
1 & 0 & 0 \\
0 & 1 & 0 \\
0 & 0 & 1 \\
0 & 0 & 0
\end{array}\right)
$$

which is equivalent to:

$$
(A)_{4 \times 4} \times(X)_{4 \times 3}=(C)_{4 \times 3}
$$

whence:

$$
(X)=(A)^{-1} \times(C) .
$$

Hence the tensors $k^{2} \hat{G}_{i j}$ and $i k \hat{H}_{i}$ are real functions of $\overline{\boldsymbol{\alpha}}$ (but not of $\overline{\boldsymbol{k}}$ ), and are obtained as two submatrices of $(X)$.

\section{Appendix B.1. Solution for the strain rate}

In terms of the Green function, the velocity field is given by:

$$
\tilde{\dot{u}}_{n}(\overline{\boldsymbol{r}})=\int_{\mathbb{R}^{3}} G_{n i}\left(\overline{\boldsymbol{r}}-\overline{\boldsymbol{r}}^{\prime}\right) f_{i}\left(\overline{\boldsymbol{r}}^{\prime}\right) \mathrm{d} \overline{\boldsymbol{r}}^{\prime} .
$$

Using equation (16), taking derivatives and integrating by parts gives:

$\tilde{\dot{u}}_{n, m}(\overline{\boldsymbol{r}})=\int_{\Omega} G_{n i, m}\left(\overline{\boldsymbol{r}}-\overline{\boldsymbol{r}}^{\prime}\right) \Delta_{i j, j}\left(\overline{\boldsymbol{r}}^{\prime}\right) \mathrm{d} \overline{\boldsymbol{r}}^{\prime}=\int_{\Omega} G_{n i, m j}\left(\overline{\boldsymbol{r}}-\overline{\boldsymbol{r}}^{\prime}\right) \Delta_{i j}\left(\overline{\boldsymbol{r}}^{\prime}\right) \mathrm{d} \overline{\boldsymbol{r}}^{\prime}$

where $\Delta_{i j}(\overline{\boldsymbol{r}})$ is defined by equation (17) and $\Omega$ is the volume of the inclusion, i.e. the domain where the fictitious force is non-zero. If $\dot{\epsilon}_{i j}^{*}$ is not a function of $\bar{r}$ and the inclusion domain is ellipsoidal and the compliance of the medium is spatially uniform (as resulting 
from tangent linearization, see equation $(5 b)$ ), the constant value of the velocity gradient inside the inclusion is:

$$
\tilde{\dot{u}}_{n, m}=\left(-\frac{1}{\Omega} \int_{\Omega} \int_{\Omega} G_{n i, m j}\left(\overline{\boldsymbol{r}}-\overline{\boldsymbol{r}}^{\prime}\right) \mathrm{d} \overline{\boldsymbol{r}} \mathrm{d} \boldsymbol{r}^{\prime}\right) L_{i j k l}^{(\mathrm{tan})} \dot{\epsilon}_{k l}^{*}=T_{m n i j} L_{i j k l}^{(\mathrm{tan})} \dot{\epsilon}_{k l}^{*} .
$$

The tensor $T_{m n i j}$ can be expressed in terms of the inverse Fourier transform $\hat{G}_{i j}(\overline{\boldsymbol{k}})$ :

$$
T_{m n i j}=-\frac{1}{\Omega} \int_{\Omega} \int_{\Omega} \int_{\mathbb{R}^{3}} \alpha_{m} \alpha_{j}\left(k^{2} \hat{\boldsymbol{G}}_{n i}(\overline{\boldsymbol{k}})\right) \exp \left(\mathrm{i} \overline{\boldsymbol{k}} \cdot\left(\overline{\boldsymbol{r}}-\overline{\boldsymbol{r}}^{\prime}\right)\right) \mathrm{d} \overline{\boldsymbol{k}} \mathrm{d} \overline{\boldsymbol{r}} \mathrm{d} \overline{\boldsymbol{r}}^{\prime}
$$

An explicit integration reduces the expression (B10) to:

$$
T_{m n i j}=\left(\frac{a b c}{4 \pi}\right) \int_{0}^{2 \pi} \int_{0}^{\pi} \frac{\alpha_{m} \alpha_{j}\left(k^{2} \hat{G}_{n i}\right)}{\rho^{3}} \sin \theta \mathrm{d} \theta \mathrm{d} \varphi
$$

where $(a, b, c)$ are the principal axes of the ellipsoid, $\theta$ and $\varphi$ are the spherical coordinates of the unit vector $\tilde{\boldsymbol{\alpha}}$, the vector $\bar{\rho}$ is defined as $\left(a \alpha_{1}, b \alpha_{2}, c \alpha_{3}\right)$ and the tensor $\left(k^{2} \hat{G}_{i j}\right)$ (a function of $\boldsymbol{\alpha}$ ) is given by (B6). The integral can be efficiently solved numerically using the Gauss quadrature technique.

\section{Appendix B.2. Solution for the pressure}

Analogous to (B7), the local pressure deviation is given by:

$$
\tilde{p}(\overline{\boldsymbol{r}})=\int_{\Omega} H_{i}\left(\overline{\boldsymbol{r}}-\overline{\boldsymbol{r}}^{\prime}\right) f_{i}\left(\overline{\boldsymbol{r}}^{\prime}\right) \mathrm{d} \overline{\boldsymbol{r}}^{\prime}=\int_{\Omega} H_{i, j}\left(\overline{\boldsymbol{r}}-\overline{\boldsymbol{r}}^{\prime}\right) \Delta_{i j}\left(\overline{\boldsymbol{r}}^{\prime}\right) \mathrm{d} \overline{\boldsymbol{r}}^{\prime} .
$$

For constant $\dot{\epsilon}_{i j}^{*}$ inside an ellipsoidal domain $\Omega$, the constant value of $\tilde{p}$ can be calculated as:

$$
\tilde{p}=\left(-\frac{1}{\Omega} \int_{\Omega} \int_{\Omega} H_{i, j}\left(\overline{\boldsymbol{r}}-\overline{\boldsymbol{r}}^{\prime}\right) \mathrm{d} \overline{\boldsymbol{r}} \mathrm{d} \overline{\boldsymbol{r}}^{\prime}\right) L_{i j k l}^{(\mathrm{tan})} \dot{\epsilon}_{k l}^{*}=P_{i j} L_{i j k l}^{(\mathrm{tan})} \dot{\epsilon}_{k l} .
$$

Using equation $(\mathrm{B} 2 b)$, the tensor $P_{i j}$ can be written as:

$$
\begin{aligned}
& P_{i j}=\frac{1}{\Omega} \int_{\Omega} \int_{\Omega} \int_{\mathbb{R}^{3}} \alpha_{j}\left(i k \hat{H}_{i}(\overline{\boldsymbol{k}})\right) \exp \left(\mathrm{i} \overline{\boldsymbol{k}} \cdot\left(\overline{\boldsymbol{r}}-\overline{\boldsymbol{r}}^{\prime}\right)\right) \mathrm{d} \overline{\boldsymbol{k}} \mathrm{d} \overline{\boldsymbol{r}} \mathrm{d} \overline{\boldsymbol{r}}^{\prime} \\
= & \left(\frac{a b c}{4 \pi}\right) \int_{0}^{2 \pi} \int_{0}^{\pi} \frac{\alpha_{j}\left(i k \hat{H}_{i}\right)}{\rho^{3}} \sin \theta \mathrm{d} \theta \mathrm{d} \varphi .
\end{aligned}
$$

The tensor $\left(i k \hat{H}_{i}\right)$ is given by (B6) and, as for (B11), this integral can be evaluated numerically using the Gauss technique.

\section{Acknowledgments}

One of the co-authors of this work, Gilles Canova, died shortly before this work was completed. The other authors mourn his untimely death and acknowledge his influence on this work in particular and on the more general field of the modelling of mechanical in general. The authors are grateful to Dr T H Holden for making available measurements of internal strains in Incoloy-800 and the experimental orientation distribution function of the material prior to publication and also to Professor S Ahzi for his valuable comments on the original version of the manuscript. 


\section{References}

[1] Lin T H 1957 Analysis of elastic and plastic strains of a face-centred cubic crystal J. Mech. Phys. Sol. 5143

[2] Kröner E 1961 Zur Plastischen des Viel-Kristalls Acta Metall. 9 155-65

[3] Budiansky B and Wu T T 1962 Theoretical prediction of plastic strains of polycrystals Proc. 4th US Congr. Appl. Mech. (New York: ASME) pp 1175-85

[4] Eshelby J D 1957 The determination of the elastic field of an ellipsoidal inclusion and related problems Proc. R. Soc. (London) A 241 376-96

[5] Hill R 1965 Continuum micro-mechanics of elastoplastic polycrystals J. Mech. Phys. Sol. 13 89-101

[6] Molinari A, Ahzi S and Kouddane R 1997 On the self-consistent modelling of elastic-plastic behavior of polycrystals Mechanics of Materials (in press)

[7] Iwakuma T and Nemat-Nasser S 1986 Finite elastic-plastic deformation of polycrystalline metals Proc. $R$. Soc. London A 407 343-76

[8] Berveiller M, Krier J, Ruppersberg H and Wagner C N J 1991 Theoretical investigation of $\psi$-splitting after plastic deformation of two-phase materials Textures and Microstructures 14-18 151-6

[9] Krier J, Ruppersberg H, Berveiller M and Lipinski P 1991 Elastic and plastic anisotropy effects on secondorder internal stresses in textures polycrystalline materials Textures and Microstructures 14-18 1147-52

[10] Van Acker K, Van Houtte P and Aernoudt E 1994 Determination of residual stresses in heavily cold deformed steel Proc. 4th Int. Conf. on Residual Stresses (ICRS-4) ed M R James (Baltimore: Society for Experimental Mechanics Inc.) pp 402-9

[11] Molinari A, Casova G R and Ahzi S 1987 A self-consistent approach of the large deformation polycrystal viscoplasticity Acta Metall. 35 2983-94

[12] Lebensohn R A and Tomé C N 1993 A self-consistent anisotropic approach for the simulation of plastic deformation and texture development of polycrystals: application to zirconium alloys Acta Metall. Mater. $412611-24$

[13] Mura T 1988 Micromechanics of Defects in Solids (Dordrecht: Martinus-Nijhoff)

[14] Hutchinson J W 1976 Bounds and self-consistent estimates for creep of polycrystalline materials Proc. $R$. Soc. London A 348 101-27

[15] Tomé C N, So C B and Woo C H 1993 Self-consistent calculation of steady-state creep and growth in textured zirconium Phil. Mag. A 67 917-30

[16] Holden T M, Holt R A, Dollong G, Powell B M and Winnegar J E 1988 Characterization of residual stresses in bent incoloy-800 tubing by neutron diffraction Met. Trans. A 19A 2207-14

[17] Leitch B W and Tomé C N 1995 The bending of incoloy-800 tubes and the resultant internal stresses Simulation of Materials Processing: Theory, Methods and Applications ed S F Shen and P R Dawson (Rotterdam: Balkema) pp 859-62

[18] Holden T M, Clarke A P and Holt R A 1997 Intergranular stresses in Incoloy-800 J. Neutron Res. 5 241-64 1996 AECL Available Report ANDI-96, COG-96-066-I

[19] Holden T M, Tomé C N, and Holt R A 1997 Experimental and theoretical studies of the superposition of intergranular and macroscopic strains in Ni-based industrial alloys Acta Metall. submitted

[20] Hutchinson J W 1970 Elastic-plastic behavior of polycrystalline metals and composites Proc. R. Soc. London A 319 247-72

[21] Turner P A and Tomé C N 1994 A study of residual stresses in Zircaloy-2 with rod texture Acta Metall. Mater. 42 4143-53

[22] Tomé C N, Holden T M, Turner P A and Lebensohn R A 1997 Interpretation of intergranular stress measurements in Monel-400 using polycrystal models Proc. 5th Int. Conf. on Residual Stresses (ICRS-5; Linkoping, Sweden) in press 